\title{
SOME CONSEQUENCES OF THE STANDARD POLYNOMIAL QING CHANG
}

(Communicated by Donald S. Passman)

ABstract. The standard polynomial of degree $m$ is the polynomial $\sum\left\{\operatorname{sign}(\rho) x_{\rho(1)} x_{\rho(2)} \cdots x_{\rho(m)} \mid \rho \in S_{m}\right\}$, where $S_{m}$ is the symmetric group on $m$ letters. We show that the polynomial

$$
\sum\left\{\operatorname{sign}(\rho \sigma) x_{\rho(1)} y_{\sigma(1)} x_{\rho(2)} y_{\sigma(2)} \cdots x_{\rho(m)} y_{\sigma(m)} \mid \rho, \sigma \in S_{m}\right\}
$$

is a consequence of the standard polynomial of degree $m$. We also show that certain polynomials of the form $\sum\left\{\operatorname{sign}(\rho) x_{\rho(1)} x_{\rho(2)} \cdots x_{\rho(n)} \mid \rho \in Q\right\}$, where $n \geq m$ and $Q$ is a suitable subset of $S_{n}$, are consequences of the standard polynomial of degree $m$.

1. Introduction. Two of the most important polynomials in the theory of polynomial identity rings are the standard polynomial and the Capelli polynomial, which are defined, respectively, for each positive integer $m$, by

$$
\begin{aligned}
\mathscr{S}_{m}\left(x_{1}, \ldots, x_{m}\right) & =\sum_{\rho \in S_{m}}(\operatorname{sign} \rho) x_{\rho(1)} x_{\rho(2)} \cdots x_{\rho(m)}, \\
\mathscr{C}_{m}\left(x_{1}, \ldots, x_{m}, y_{1}, \ldots, y_{m}\right) & =\sum_{\rho \in S_{m}}(\operatorname{sign} \rho) x_{\rho(1)} y_{1} x_{\rho(2)} y_{2} \cdots x_{\rho(m)} y_{m} .
\end{aligned}
$$

The Amitsur-Levitzki Theorem says that $\mathscr{S}_{2 n}$ is a polynomial identity for $M_{n}(K)$, the ring of $n \times n$ matrices over a field $K$, but $\mathscr{S}_{2 n-1}$ is not. Razmyslov has shown that $\mathscr{C}_{n^{2}+1}$ is a polynomial identity for $M_{n}(K)$, but $\mathscr{C}_{n^{2}}$ is not.

This paper was motivated by a question of Formanek. Define the double Capelli polynomial by

$$
\mathscr{D}_{m}\left(x_{1}, \ldots, x_{m}, y_{1}, \ldots, y_{m}\right)=\sum_{\rho, \sigma \in S_{m}}(\operatorname{sign} \rho \sigma) x_{\rho(1)} y_{\sigma(1)} \ldots x_{\rho(m)} y_{\sigma(m)} .
$$

Formanek pointed out that $\mathscr{D}_{2 n-1}$ is not a polynomial identity for $M_{n}(K)$ and asked for the least integer $m$ such that $\mathscr{D}_{m}$ is a polynomial identity for $M_{n}(K)$. We will show (Theorem 2) that $m=2 n$ is the least such integer. This result will be a corollary of our main result (Theorem 1), which says that certain polynomials, one of which is $\mathscr{D}_{m}$, are consequences of the standard polynomial $\mathscr{S}_{m}$. (A polynomial $\mathscr{F}$ is a consequence of a polynomial $\mathscr{G}$ if it lies in the $T$-ideal generated by $\mathscr{G}$.)

These polynomials are defined as follows. Let $x_{1}, \ldots, x_{m}, y_{1}, \ldots, y_{t}$ be noncommuting variables over $K$, and let $w_{1}, \ldots, w_{u}$ be monomials in $y_{1}, \ldots, y_{t}$ such that $w_{1} \ldots w_{u}$ is a reordering of $y_{1} \ldots y_{t}$. Define a polynomial

$$
\mathscr{F}=\mathscr{F}\left(x_{1}, \ldots, x_{m}, y_{1}, \ldots, y_{t} \mid w_{1}, \ldots, w_{u}\right)
$$

Received by the editors November 24, 1987.

1980 Mathematics Subject Classification (1985 Revision). Primary 16A38.

Key words and phrases. Polynomial identity, standard polynomial, Capelli polynomial. 
by

$$
\begin{aligned}
& \mathscr{F}=\sum(\operatorname{sign} \mu) x_{\rho(1)} \ldots x_{\rho\left(n_{1}\right)} w_{\sigma(1)} x_{\rho\left(n_{1}+1\right)} \cdots x_{\rho\left(n_{1}+n_{2}\right)} w_{\sigma(2)} x_{\rho\left(n_{1}+n_{2}+1\right)} \\
& \ldots x_{\rho\left(n_{1}+\cdots+n_{u}\right)} w_{\sigma(u)} x_{\rho\left(n_{1}+\cdots+n_{u}+1\right)} \ldots x_{\rho\left(n_{1}+\cdots+n_{u+1}\right)} \text {, }
\end{aligned}
$$

where the sum is over all $\rho \in S_{m}, \sigma \in S_{u}$, and all choices of integers $n_{1}, \ldots, n_{u} \geq 1$, $n_{u+1} \geq 0$, such that $n_{1}+\cdots+n_{u+1}=m$, and the sign attached to each such monomial $\mu$ is \pm 1 according to whether $\mu$ is an even or an odd permutation of the "underlying" variables $x_{1}, \ldots, x_{m}, y_{1}, \ldots, y_{t}$. Theorem 1 states that $\mathscr{F}$ is a consequence of the standard polynomial $\mathscr{S}_{m}$ of degree $m$.

A few comments about the polynomial $\mathscr{F}$ are in order. First, it is a homogeneous multilinear polynomial in the variables $x_{1}, \ldots, x_{m}, y_{1}, \ldots, y_{t}$, and might more properly be written $\mathscr{F}_{\pi}\left(x_{1}, \ldots, x_{m}, y_{1}, \ldots, y_{t}\right)$, where $\pi=\left(w_{1}, \ldots, w_{u}\right)$ is a partition of a reordering of the product $y_{1} \ldots y_{t}$ into $u$ subwords. Second, $\mathscr{F}$ is a summand of the standard polynomial $\mathscr{S}_{m+t}$, in the sense that

$$
\mathscr{F}=\sum_{\rho \in Q}(\operatorname{sign} \rho) z_{\rho(1)} \cdots z_{\rho(m+t)},
$$

where $\left\{x_{1}, \ldots, x_{m}, y_{1}, \ldots, y_{t}\right\}=\left\{z_{1}, \ldots, z_{m+t}\right\}$ and $Q$ is a certain subset of $S_{m+t}$. Third, the sign attached to a monomial $\mu$ depends only on the parity of the lengths of $w_{1}, \ldots, w_{u}$. Fourth, the condition on $n_{1}, \ldots, n_{u+1}$ means that the sum is precisely over those monomials in $x_{1}, \ldots, x_{m}, w_{1}, \ldots, x_{u}$ having the property that each $w_{i}$ is immediately preceded by some $x_{j}$. This point is important in the proof of the main theorem.

\section{Proofs of the theorems.}

THEOREM 1. $\mathscr{F}\left(x_{1}, \ldots, x_{m}, y_{1}, \ldots, y_{t} \mid w_{1}, \ldots, w_{u}\right)$ is a consequence of the standard polynomial $\mathscr{S}_{m}$ of degree $m$.

PROOF. The proof is by induction on the pair $(t, u)$ associated with $\mathscr{F}$, where $\left(t_{1}, u_{1}\right)<(t, u)$ if $t_{1}<t$, or $t_{1}=t$ and $u_{1}<u$.

The induction begins with $(1,1)$, when $w_{1}=y_{1}$. Then

$$
\mathscr{S}_{m+1}\left(x_{1}, \ldots, x_{m}, y_{1}\right)=(-1)^{m} y_{1} \mathscr{S}_{m}\left(x_{1}, \ldots, x_{m}\right)+\mathscr{F}\left(x_{1}, \ldots, x_{m}, y_{1} \mid w_{1}\right),
$$

since $\mathscr{F}\left(x_{1}, \ldots, x_{m}, y_{1} \mid w_{1}\right)$ is the sum, with the right sign, of all the monomials in $\mathscr{S}_{m+1}\left(x_{1}, \ldots, x_{m}, y_{1}\right)$ in which $y_{1}$ is immediately preceded by some $x_{i}$. Since $\mathscr{S}_{m+1}$ is a consequence of $\mathscr{S}_{m},(1)$ shows that $\mathscr{F}\left(x_{1}, \ldots, x_{m}, y_{1} \mid w_{1}\right)$ is also a consequence of $\mathscr{S}_{m}$.

For the inductive step, assume that $\mathscr{F}\left(x_{1}, \ldots, x_{m}, y_{1}, \ldots, y_{t_{1}} \mid w_{1}, \ldots, w_{u_{1}}\right)$ is a consequence of $\mathscr{S}_{m}$ whenever $\left(t_{1}, u_{1}\right)<(t, u)$. There are three cases, which are not mutually exclusive.

Case 1. Some monomial $w_{i}$ has length $\geq 3$ in terms of $y_{1}, \ldots, y_{t}$. To avoid cumbersome notation, we assume that the monomial in question is $w_{u}=* \cdots *$ $y_{t-2} y_{t-1} y_{t}$. Now

$$
\begin{aligned}
& \mathscr{F}\left(x_{1}, \ldots, x_{m}, y_{1}, \ldots, y_{t-3}, y_{t-2}, y_{t-1}, y_{t} \mid w_{1}, \ldots, w_{u}\right) \\
& \quad=\mathscr{F}\left(x_{1}, \ldots, x_{m}, y_{1}, \ldots, y_{t-3},\left(y_{t-2} y_{t-1} y_{t}\right) \mid w_{1}, \ldots, w_{u}\right),
\end{aligned}
$$

since both functions are sums of exactly the same monomials, with the same signs. However, the pair $(t, u)$ is associated with the first polynomial, while the pair 
$(t-2, u)$ is associated with the second polynomial. Thus the second polynomial in (2) is a consequence of $\mathscr{S}_{m}$ by the inductive hypothesis, and so the first polynomial in (2) is also a consequence of $\mathscr{S}_{m}$.

Case 2 . Some monomial $w_{i}$ has length exactly 2 . Again, for notational simplicity, we assume that the monomial is $w_{u}=y_{t-1} y_{t}$. Consider the equation

(3)

$$
\begin{aligned}
\mathscr{F}\left(x_{1}, \ldots, x_{m}, y_{1}, \ldots, y_{t} \mid w_{1}, \ldots, w_{u}\right) \\
=\sum_{1 \leq i \leq m} \mathscr{F}\left(x_{1}, \ldots, x_{i-1}, x_{i} w_{u}, x_{i+1}, \ldots, x_{m}, y_{1}, \ldots, y_{t-2} \mid w_{1}, \ldots, w_{u-1}\right) \\
\quad-\sum_{1 \leq j \leq u} \mathscr{F}\left(x_{1}, \ldots, x_{m}, y_{1}, \ldots, y_{t} \mid w_{1}, \ldots, w_{j-1}, w_{u} w_{j}, w_{j+1}, \ldots, w_{u-1}\right) .
\end{aligned}
$$

To see that the above equation is valid, note that when the first function is written as an alternating sum of monomials, $w_{u}$ is immediately preceded in each monomial by some $x_{i}$ and thus occurs exactly once, with the proper sign, in the first summation of the right-hand side of the equation. Conversely, every monomial in this first summation occurs with the proper sign in the first function, except those monomials where $x_{i} w_{u}$ immediately precedes some $w_{j}$. Subtracting the final summation eliminates these monomials and verifies equation (3).

The $\mathscr{F}$ 's in the first summation have associated pair $(t-2, u-1)$ and the $\mathscr{F}$ 's in the second summation have associated pair $(t, u-1)$. Hence both are consequences of $\mathscr{S}_{m}$, by the inductive hypothesis, and so $\mathscr{F}\left(x_{1}, \ldots, x_{m}, y_{1}, \ldots, y_{t} \mid w_{1}, \ldots, w_{u}\right)$ is also a consequence of $\mathscr{S}_{m}$.

Case 3. All the $w_{i}$ 's have length 1 . Then, up to a change of sign, we may suppose that $w_{1}=y_{1}, \ldots, w_{t}=y_{t}$. Consider $\mathscr{S}_{m+t}\left(x_{1}, \ldots, x_{m}, y_{1}, \ldots, y_{t}\right)$, the standard polynomial. When it is expanded, every monomial in the expansion has the form

$$
\mu_{0}(y) \nu_{1}(x) \mu_{1}(y) \nu_{2}(x) \mu_{2}(y) \cdots \nu_{r}(x) \mu_{r}(y) \nu_{r+1}(x)
$$

where $r \geq 0, \mu_{0}(y), \ldots, \mu_{r}(y)$ are monomials in $y_{1}, \ldots, y_{t}, \nu_{1}(x), \ldots, \nu_{r+1}(x)$ are monomials in $x_{1}, \ldots, x_{m}$, and all these monomials are nontrivial except possibly $\mu_{0}(y)$ and $v_{r+1}(x)$. The monomials with the same $\mu_{0}(y)$ and unordered set $\left\{\mu_{1}(y), \ldots, \mu_{r}(y)\right\}$ together make a contribution to $\mathscr{S}_{m+t}$ equal to

$$
\pm \mu_{0}(y) \mathscr{F}\left(x_{1}, \ldots, x_{m}, y_{i_{1}}, \ldots, y_{i_{s}} \mid \mu_{1}(y), \ldots, \mu_{r}(y)\right) \text {. }
$$

One such summand of $\mathscr{S}_{m+t}$ is $\mathscr{F}\left(x_{1}, \ldots, x_{m}, y_{1}, \ldots, y_{t} \mid y_{1}, \ldots, y_{t}\right)$, which has associated pair $(t, t)$, but all of the remaining $\mathscr{F}$ 's in the summation have associated pair $(s, r)<(t, t)$, and thus are consequences of $\mathscr{S}_{m}$, by the inductive hypothesis. Thus $\mathscr{F}\left(x_{1}, \ldots, x_{m}, y_{1}, \ldots, y_{t} \mid y_{1}, \ldots, y_{t}\right)$ is also a consequence of $\mathscr{S}_{m}$, completing the induction.

The answer to Formanek's question is a corollary to Theorem 1.

THEOREM 2. Let

$$
\mathscr{D}_{m}\left(x_{1}, \ldots, x_{m}, y_{1}, \ldots, y_{m}\right)=\sum_{\rho, \sigma \in S_{m}}(\operatorname{sign} \rho \sigma) x_{\rho(1)} y_{\sigma(1)} \cdots x_{\rho(m)} y_{\sigma(m)}
$$

Then $\mathscr{D}_{2 n}$ is a polynomial identity for $M_{n}(K)$, the ring of $n \times n$ matrices over a field $K$, but $\mathscr{D}_{2 n-1}$ is not. 
PROOF. $\mathscr{D}_{2 n}\left(x_{1}, \ldots, x_{2 n}, y_{1}, \ldots, y_{2 n}\right)=\mathscr{F}\left(x_{1}, \ldots, x_{2 n}, y_{1}, \ldots, y_{2 n} \mid y_{1}, \ldots, y_{2 n}\right)$. Since $\mathscr{F}$ is a consequence of $\mathscr{S}_{2 n}$ and $\mathscr{S}_{2 n}$ is a polynomial identity for $M_{n}(K)$ (Amitsur-Levitzki Theorem), it follows that $\mathscr{D}_{2 n}$ is a polynomial identity for $M_{n}(K)$.

To see that $\mathscr{D}_{2 n-1}$ is not a polynomial identity for $M_{n}(K)$, consider the substitution

$$
\begin{aligned}
\left(u_{1}, \ldots, u_{2 n-1}\right) & =\left(e_{11}, e_{22}, \ldots, e_{n n}, e_{n n-1}, e_{n-1 n-2}, \ldots, e_{21}\right) \\
\left(v_{1}, \ldots, v_{2 n-1}\right) & =\left(e_{12}, e_{23}, \ldots, e_{n-1 n}, e_{n n}, e_{n-1 n-1}, \ldots, e_{11}\right),
\end{aligned}
$$

where the $e_{i j}$ are the standard matrix units. The only nonzero monomials in $\mathscr{D}_{2 n-1}\left(u_{i}, v_{i}\right)$ are the $2 n-1$ even cyclic permutations of $u_{1} v_{1} \ldots u_{2 n-1} v_{2 n-1}$, and they all have sign +1 . Thus

$$
\begin{aligned}
\mathscr{D}_{2 n-1}\left(u_{i}, v_{i}\right) & =e_{11}+e_{22}+\cdots+e_{n n}+e_{n n}+\cdots+e_{22} \\
& =2 I-e_{11} .
\end{aligned}
$$

Acknowledgement. I am grateful to Professor Edward Formanek who gave me advice and revised the whole paper.

ADDED IN PROOF: Recently I was told that Professor A. Giambruno and Professor S. K. Sehgal proved the result of Theorem 2.

\section{REFERENCES}

1. S. A. Amitsur, Alternating identities, Ring Theory (S. K. Jain editor), Proceedings of the Ohio Univ. Conference., pp. 1-14.

2. __ Polynomial identities, Israel J. Math. 19 (1974), 183-199.

3. E. Formanek, The polynomial identities of matrices, Contemp. Math., vol. 32, Amer. Math. Soc., Providence, R.I., 1982, pp. 41-79.

4. N. Jacobson, PI-algebras. An introduction, Lecture Notes in Math., vol. 441, Springer-Verlag, Berlin and New York, 1975.

5. L. H. Rowen, Polynomial identities in ring theory, Academic Press, New York, 1980.

Department of Mathematics, Pennsylvania State University, University Park, PenNSylVania 16802

Department of Mathematics, Hubei University, Wuhan, People's Republic OF CHINA 